the introduction of comparatively small masses on the earth's surface would have no perceptible effect on the gravitational field, $s$ may be taken as constant in any experiments on the earth's surface.

Dr. P. E. Shaw (NATURE, March 29) argues that my hypothesis involves a violation of the conservation of energy. To avoid the difficulty of perpetual motion I would suggest making $\partial Q / \partial r$ positive instead of negative. This will not alter the expression for $F$, but the turning moment on Dr. Shaw's disc will then bring it to rest.

Newcastle-upon-Tyne.

\section{THERMIONIC DETECTORS IN WIRELESS TELEGRAPHY AND TELEPHONY.}

$r \mathrm{HE}$ arts of wireless telegraphy and telephony involve the use in the receiving circuit of some device named a detector, which is sensitive to electric oscillations of very high frequency. In the earliest years of radiotelegraphy the appliance used was the so-called coherer, in which a small mass of metallic filings or an imperfect contact between two pieces of metal was converted into a better conductor by the passage through it of the high-frequency oscillations. All the various forms of coherer have now been abandoned and are no longer used as detectors. In modern radiotelegraphy, so far as regards the spark or damped-wave system, only three types of detector are at present in practical use. The first of these is the magnetic detector, chiefly the rotating band form, invented by Marconi; the second type is some form of rectifying contact or crystal, such as the carborundum detector due to Dunwoody, or the zincite-chalcopyrite rectifier of Pickard; and the third is some modification of the thermionic detector, or Fleming oscillation valve.

In the magnetic detector the electric oscillations to be detected are caused to circulate round a magnetised iron wire and alter its magnetic permeability or hysteresis in such a fashion as to create a sudden change in the magnetisation of the iron. This in turn is made to create an induced electric current in a second coil and reveal itself by a sound made in a telephone in series with that coil. The rectifying contacts or crystals depend upon the fact that a contact of small surface between certain substances, generally crystalline, has a greater electric conductivity in one direction than in the other. Hence, if such a contact as that, for example, between a fragment of zincite or native oxide of zinc and a piece of chalcopyrite or copper pyrites is traversed by a train of electric oscillations, these will be converted into a movement of electricity chiefly in one direction. Accordingly, if a rapid sequence of such oscillations passes through such rectifying contact placed in series with a telephone receiver, the latter will be traversed by a series of intermittent gushes of electricity in the same direction, and will emit a sound the pitch of which is determined by the group frequency of the oscillations. A very commonly used rectifying contact is a crystal of carborundum, or carbide of silicon, held between metal clips. Although this rectifying property of certain contacts and crystals has been much studied, the reasons for it are not yet fully elucidated, but it is probably connected with the thermoelectric properties of the materials.

The third type of detector is the thermionic detector first suggested and used by Dr. J. A. Fleming, of University College, London. The construction and mode of operation of this form of detector may be briefly described as follows :It had been known for many years prior to the advent of radiotelegraphy that the electric conductivity of a highly rarefied gas was greatly determined by the temperature of the negative electrode by which the current left the exhausted vessel containing it. It had been found by Hittorf and also by Elster and Geitel that if the negative electrode was a platinum wire which could be rendered incandescent, the conductivity of the highly rarefied gas was greatly increased. The emission of positive and of negative ions from incandescent solids in vacuo had been studied particularly by Elster and Geitel, beginning in I 880 .

In 1884 Edison made known an interesting fact connected with carbon-filament glow-lamps. He sealed into the bulb of one of his bamboo-filament lamps a metal plate placed between the legs of the horseshoe-shaped filament, the said plate being carried on a platinum wire sealed through the glass bulb. He found that when the filament was rendered incandescent by a continuous current, a galvanometer connected between the terminal of the plate and the external negative terminal of the filament indicated no current, but that if connected between the plate and the positive filament terminal, it showed a current of a few milliamperes. Edison gave no explanation of this, nor did he make any application of the discovery. He supplied a certain number of lamps made with middle plates to the late Sir William Preece, and the latter communicated to the Royal Society in 1885 a paper describing various experiments with these lamps. This "Edison effect" was more completely examined by Dr. J. A. Fleming in researches described by him in papers to the Royal Society in $\mathbf{r} 890$ and to the Physical Society in I 896. Dr. Fleming showed that the effect was in some way due to the scattering of particles charged with negative electricity from the hot filament, and that it could be prevented by enclosing the negative leg of the carbon in a glass tube, or placing a sheet of mica between the carbon and the plate. He also proved, as Elster and Geitel had done in another way, that a vacuum tube having two carbon filaments as electrodes had a very large conductivity for small voltages when the negative electrode was made incandescent.

It was not until I899, when Sir J. J. Thomson announced his epoch-making discovery of electrons, or corpuscles smaller than atoms, carrying a negative charge, that it was clearly recognised that incandescent solids in high vacua emit electric ions, some positive and some negative.

This electronic emission from hot bodies has 
been very fully investigated by Prof. O. W Richardson, who has collected most of the known facts in an excellent manual on the subject. None of the investigators of this subject made any practical application of this knowledge until it occurred to Dr. Fleming in 1904 to employ an incandescent electric lamp having one or more plates or cylinders of metal sealed into the bulb as a means of detecting high-frequency electric
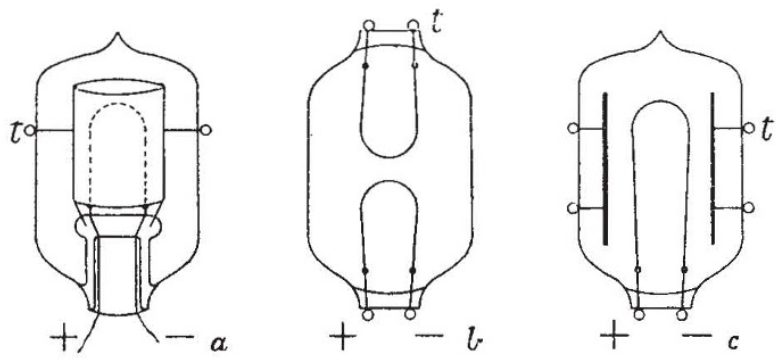

.1.-Various forms of Fleming oscillation valve or thermionic detector used in wireless telegraphy.

oscillations, as used in radiotelegraphy. Accordingly he constructed such electric glow-lamps with carbon filaments and a metal plate or cylinder surrounding, but not touching, the filament, the said cylinder being attached to a platinum wire sealed through the bulb (see Fig. I). $\mathrm{He}$ employed this device as follows:- The carbon filament in the lamp $\mathrm{O}$ (see Fig. 2) is rendered in-

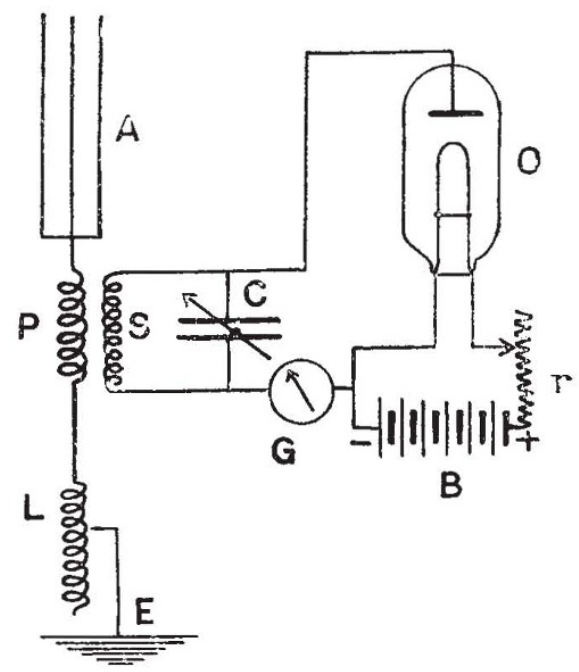

FIG. 2.-One mode of employing the oscillation valve as a detector in a radiotelegraphic receiving circuit. A, antenna; $\mathrm{PS}$, oscillation transformer; C, condenser; O, oscillation valve; $\mathrm{B}$, battery ; $\mathrm{G}$, currentdetecting instrument.

candescent by a suitable battery of storage cells, B ; most usually a 12 -volt or 4 -volt filament is employed. A circuit is formed external to the bulb by connecting the metal plate or cylinder to the negative terminal of the lamp. In this circuit is placed a current-detecting instrument, such as a galvanometer, $\mathrm{G}$, or a telephone. In the circuit is also inserted the secondary circuit of an oscilla- tion transformer, $\mathrm{P}, \mathrm{S}$, the terminals of which are closed by a condenser, C (see Fig. 2).

If electric oscillations are created in the above circuit, the alternations of current are rectified; that is to say, a unidirectional current flows through the galvanometer or telephone. The highly vacuous space between the incandescent filament and the metal cylinder inside the bulb possesses a unilateral conductivity. When the filament is at a very high temperature negative electricity can pass from the filament to the plate, but not in the opposite direction. Hence the device acts as a valve and was called by Dr. Fleming an oscillation valve. Another way of viewing the effect is as follows:-When the electric oscillations take place through the condenser, the plate or cylinder in the bulb tends to become charged alternately positive and negative. The incandescent filament is continuously emitting negative ions or electrons, and these at once discharge any positive charge on the metal plate, whereas they do not discharge a negative charge. There is, therefore, a continuous movement of positive electricity to the plate from outside the lamp. If the electric oscillations are in trains of damped groups, then the effect is to convert them into gushes of electricity in one direction which pass through the telephone. If these groups come at the rate of several hundred per second the telephone receiver emits a continuous sound of corresponding pitch, and if the groups are cut up into Morse signals at the sending end, the listener at the telephone hears these signals as long and short sounds.

An electric incandescent lamp with metal plates, grids, or cylinders in the bulb is now called a thermionic detector, because it serves to rectify and render detectable by a galvanometer or telephone receiver the feeble electric oscillations used in wireless telegraphy or telephony. It depends for its action upon the emission by the incandescent filament of electrons, or thermions as they are termed.

Dr. Fleming found that a tungsten filament was of special utility for this purpose. The thermionic receiver has great advantages in that it is not injured or put out of adjustment, like crystal detectors, by powerful electric oscillations or atmospheric discharges acting on its receiving circuits.

In some of Dr. Fleming's experiments he employed an incandescent lamp with two plates sealed into the bulb carried on separate terminals. An illustration of such a double-anode or twoelement valve was given by him in a paper published in the Proceedings of the Royal Society early in 1905 (see $c$, Fig. I). The new thermionic detector naturally attracted the attention of radiotelegraphists, and amongst others of Dr. Lee de Forest in the United States. After adopting the detector in substantially the same form, Dr. de Forest patented in 1907 a modification in which the two metal electrodes were sealed into the vacuous bulb, in addition to the metallic or

NO. 2475, VOL. 99] 
carbon filament to be rendered incandescent. One of these electrodes was in the form of a plate, and the other of a grid or zigzag of wire interposed between the filament and the plate. In using this double-plate thermionic detector, Dr. de Forest connected the grid terminal to one side of the receiving circuit condenser, and the negative terminal of the filament to the other side of the same condenser; but, instead of inserting the telephone or current-detecting instrument in this grid circuit, he included it in a separate external circuit connecting the plate with the filament, and placed in this circuit also a battery with negative pole connected to the filament (see Fig. 3).

Dr. de Forest calied this arrangement an audion, and maintained that the physical action was different from that of the Fleming valve, though valves with two anode plates had already been in use for certain experiments. It has been shown, however, to be essentially the same. It is clear that the performance of the audion as a radiotelegraphic detector depends entirely upon the thermionic emission from the incandescent flament. It has been demonstrated by Dr. E. H. Armstrong in a paper in the Proceedings of the Institute of Radio-Engineers for September, I9I5, that the physical actions taking place in the grid circuit of Dr. de Forest's audion are precisely the same as in those in the Fleming valve.

The thermionic emission of negative ions causes the grid to become negatively charged. On the other hand, the battery in the external circuit connected to the plate sends a thermionic current through the vacuous space between the filament and the plate inside the bulb, in virtue of the incandescence of the filament or negative electrode. This current flows also through the telephone or current-detecting appliance. When the grid becomes negatively charged, due to the rectification of electric oscillations impressed upon the grid circuit, it reduces the thermionic current flowing between the filament and the plate, and therefore varies the current through the telephone. The physical actions which contribute to the operation are therefore all dependent upon the thermionic emission from the filament and upon the increased unilateral conductivity of a highly rarefied gas or vacuous space when the cathode or negative electrode is rendered incandescent.

This action is not necessarily dependent upon the presence of any residual gas in the bulb, because even in a highly perfect vacuum the electronic emission from the incandescent filament would take place.

The double-anode Fleming valve, or the valve with grid and plate, called an audion, has the property that an amplification of current variation can be produced by it.

Thus, if the grid-plate thermionic detector is arranged as in Fig. 3, feeble electric oscillations taking place in the grid external circuit can be made to produce large variations in the continuous current flowing in the external plate circuit.

Moreover, by connecting two or more such double-anode thermionic detectors in series, the current in the plate circuit of one, acting inductively on the grid circuit of the next, enables a double amplification to be produced.

Furthermore, such double-anode thermionic valves can be used as generators of electric oscillations by inductively connecting through a suitable transformer the grid and plate circuits $g$ and $h$

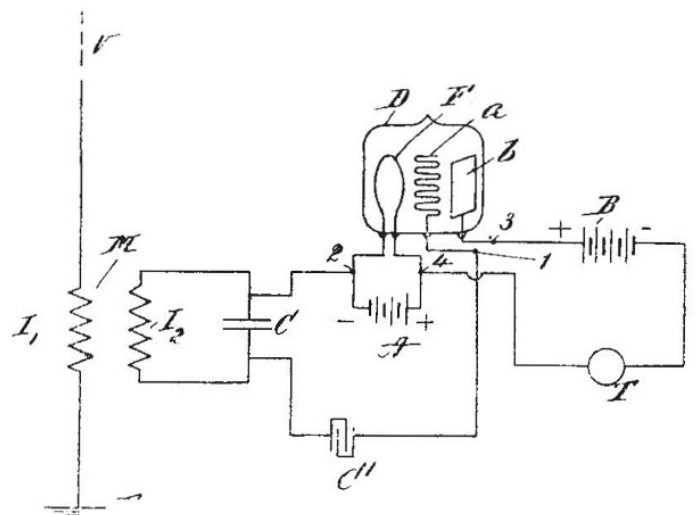

FIt. 3.-De Forest audion or form of thermionic detector. D, bulb of glow-lamp: $F$, incandescent filament ; $a$, grid; $b$, plate; $T$, telephone ; $\mathrm{A}, \mathrm{B}$, batteries ; $\mathrm{C}^{\prime}, \mathrm{C}^{\prime \prime}$, condensers.

of one and the same bulb V (see Fig. 4). The arrangement then acts as follows :-Feeble electric oscillations set up in the external plate circuit by any means create induced oscillations in the grid circuit, and the latter sustain and enhance the former, the energy to create these enhanced oscillations coming from the battery in the plate circuit.

The process exactly resembles that in which a

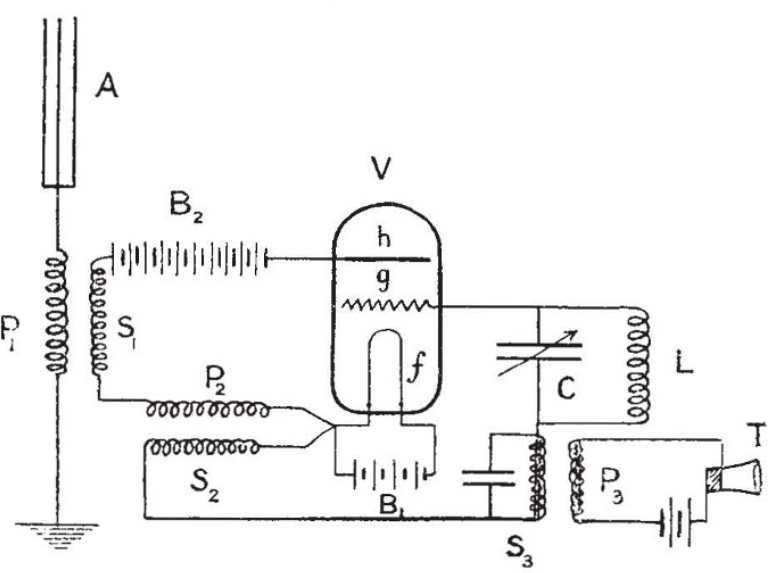

Fici. +--Mode of using double-anode valve or thermionic detector as at generator for electric oscillations.

Bell telephone receiver in circuit with a battery and carbon microphone transmitter emits a continuous musical note when the diaphragm of the receiver is held near that of the transmitter. Feeble vibrations are set up in the microphone diaphragm by noises in the room, and these vary the current through the telephone receiver, and the sound so emitted keeps the transmitter diaphragm in motion.

NO. 2475, VOL. 99] 
Again, the double-anode valve can be used as a telephonic relay in ordinary telephony to magnify and repeat sounds.

The oscillation valve is not simply a detector; it is a quantitative detector, and hence has been extensively used as a receiver in all experiments in wireless telephony. In fact, most of the successful long-distance experiments in radiotelephony have been conducted by it. For when so used it rectifies the continuous high-frequency oscillations in the receiving circuit into a direct current. Hence the variations in amplitude in these oscillations which are produced by the microphone in the transmitter circuits make themselves evident as variations in the rectified current which flows through the telephone receiver, and these reproduce the sounds of the speech made to the microphone in the transmitter. This thermionic detector promises, therefore, to be of great use in the solution of the problem of radiotelephony, as well as that of repeating or relaying ordinary telephonic currents.

\section{THE INDIAN SCIENCE CONGRESS.}

THE Indian Science Congress held its fourth annual meeting in Bangalore on January Io and the three following days, under the presidency of Sir Alfred Bourne, F.R.S. The six sectionsthose, namely, of Mathematics and Physics, Chemistry, Agriculture, Botany, Zoology, and Geology-met in the mornings, and in all seventytwo papers were read. It is obviously impossible, in the space available for this notice, to give an account of the work of the various sections or even to enumerate the papers, but certain points in connection with the present meeting are deserving of mention. Two of the sectional presidents departed from the usual custom in giving addresses on general topics. In the Mathematics and Physics Section the Rev. Dr. Mackichan referred to the great value of early Indian contributions to mathematics, both pure and applied, but deprecated the suggestion put forward by some enthusiasts that there was no scientific truth of importance that could not be traced in the ancient Hindu scriptures. The other address, given to the Chemistry Section, is referred to below.

There was a comparatively large proportion-about one-third of the total number-of papers dealing with the application of science to particular industrial problems. The increase in the number of papers of this kind is undoubtedly due to war conditions, which have stimulated industrial enterprise in many parts of India. The papers on industrial science read at meetings of the Congress represent but a small part of the work which is being carried on in different parts of the country; those on pure science, on the other hand, record very nearly the whole of what is being done in Indian colleges, and one cannot help noticing their fewness. The causes of the paucity of research work were examined by Dr. J. L. Simonsen in his presidential address to the NO. 2475 , VOL. 99]
Chemistry Section. Lack of proper training in past years, understaffing of colleges-resulting in a man's whole time being taken up by routine work-and inadequate pay in subordinate grades of the teaching profession were mentioned as among the most important; and to these must be added the absence of the research atmosphere that is so marked a feature of the larger English educational centres. The Indian Science Congress constitutes at present the only means of remedying this situation effectively. It can, through the proper official channels, direct the attention of the Imperial and local Governments to those defects of the present system which it is in their power to remedy; it can also provide once a year the research atmosphere and facilities for discussion and criticism which are lacking in the colleges, partly because the great distances which separate them make the personal exchange of ideas almost impossible, and partly because, excepting a few agricultural research stations, not more than one or two men are working at the same subject in any one place.

Although the actual amount of research in pure science is small, it is large when compared with what was being done four years ago. At the first meeting of the Congress in 1914 only twenty papers were read: the number this year had increased to seventy-two. This year, too, a new rule was in force, making it necessary for authors to submit their papers to a referee. While in some of the sections-that of Zoology, for example-the quality of the papers was excellent, this is not true of all. There were a number of papers from a certain quarter that appear to have been inspired by a determination to produce the maximum quantity of "research" in a given time. Work of this type falls into its proper place in the course of the discussion and criticism which take place in the sectional meetings, and there is no doubt that the Congress is doing a good deal towards setting up a higher standard of work than exists at present.

An interesting discussion took place, under the chairmanship of Sir Sydney Burrard, F.R.S., on scientific libraries in India, following some suggestions which had been made to the effect that research work in India was sometimes hampered by inability to obtain references. It appeared from the contributions to this discussion that the difficulty was felt chiefly by zoologists, to whom plates and diagrams were frequently of greater importance than the text of a paper (which could always be copied and sent by post). But the general feeling was that any lack of library facilities in India could scarcely be considered a contributory factor in hindering research, and that the existing needs would be adequately met by the preparation of a catalogue showing the periodicals available in different places and the rules under which they could be lent or copied.

The remaining activities of the Congress may be briefly mentioned. Three public lectures were delivered, and were attended by large audiences. The first was by Mr. C. Michie Smith on "The 